University of Wollongong

Research Online

Faculty of Engineering and Information

Faculty of Engineering and Information

Sciences - Papers: Part A

Sciences

$1-1-2012$

\title{
Enhanced optical conductance in graphene superlattice due to anisotropic band dispersion
}

Yee Sin Ang

University of Wollongong, ysa190@uowmail.edu.au

Chao Zhang

University of Wollongong, czhang@uow.edu.au

Follow this and additional works at: https://ro.uow.edu.au/eispapers

Research Online is the open access institutional repository for the University of Wollongong. For further information contact the UOW Library: research-pubs@uow.edu.au 


\title{
Enhanced optical conductance in graphene superlattice due to anisotropic band dispersion
}

\author{
Abstract \\ The optical response of a Kronig-Penney type graphene superlattice is investigated. When an external \\ field is applied along the periodicity of the superlattice, the total optical response of the graphene \\ superlattice is enhanced due to the formation of anisotropic Dirac fermions. Such anisotropy tunes up the \\ total optical spectra while maintaining the same critical electric field regardless of the degree of \\ anisotropy. The optical conductance of anisotropic Dirac fermions exhibits two contrasting behaviours: (i) \\ inversely proportional to the anisotropy and (ii) directly proportional to the anisotropy, depending on the \\ direction of the external field. Interestingly, the anisotropy-induced optical conductance enhancement \\ also occurs in gapped graphene with band structure anisotropy. This suggests that the enhanced \\ electron-photon couplings in the presence of anisotropy is a general feature of the relativistic nature of \\ the Dirac fermions in both massless and massive form. It is also revealed that the strong optical \\ nonlinearity is a consequence of the relativistic nature of the Dirac fermions and the Dirac cone isotropy is \\ not required. (c) 2012 IOP Publishing Ltd.
}

\section{Keywords}

conductance, graphene, superlattice, due, optical, anisotropic, enhanced, band, dispersion

\section{Publication Details}

Ang, Y. Sin. \& Zhang, C. (2012). Enhanced optical conductance in graphene superlattice due to anisotropic band dispersion. Journal of Physics D: Applied Physics, 45 (39), 1-5. 
Enhanced optical conductance in graphene superlattice due to anisotropic band dispersion

This article has been downloaded from IOPscience. Please scroll down to see the full text article.

2012 J. Phys. D: Appl. Phys. 45395303

(http://iopscience.iop.org/0022-3727/45/39/395303)

View the table of contents for this issue, or go to the journal homepage for more

Download details:

IP Address: 130.130.37.84

The article was downloaded on 03/12/2012 at 04:18

Please note that terms and conditions apply. 


\title{
Enhanced optical conductance in graphene superlattice due to anisotropic band dispersion
}

\author{
Yee Sin Ang and C Zhang \\ School of Engineering Physics, University of Wollongong, New South Wales, 2522, Australia \\ E-mail: czhang@uow.edu.au
}

Received 10 May 2012, in final form 23 August 2012

Published 17 September 2012

Online at stacks.iop.org/JPhysD/45/395303

\begin{abstract}
The optical response of a Kronig-Penney type graphene superlattice is investigated. When an external field is applied along the periodicity of the superlattice, the total optical response of the graphene superlattice is enhanced due to the formation of anisotropic Dirac fermions. Such anisotropy tunes up the total optical spectra while maintaining the same critical electric field regardless of the degree of anisotropy. The optical conductance of anisotropic Dirac fermions exhibits two contrasting behaviours: (i) inversely proportional to the anisotropy and (ii) directly proportional to the anisotropy, depending on the direction of the external field. Interestingly, the anisotropy-induced optical conductance enhancement also occurs in gapped graphene with band structure anisotropy. This suggests that the enhanced electron-photon couplings in the presence of anisotropy is a general feature of the relativistic nature of the Dirac fermions in both massless and massive form. It is also revealed that the strong optical nonlinearity is a consequence of the relativistic nature of the Dirac fermions and the Dirac cone isotropy is not required.
\end{abstract}

(Some figures may appear in colour only in the online journal)

\section{Introduction}

The K-point electrons in graphene are analogue to massless Dirac fermions travelling with a reduced 'light speed' of $v_{\mathrm{F}}=10^{6} \mathrm{~m} \mathrm{~s}^{-1}$ and is perfectly isotropic in all directions $[1,2]$. This peculiar quasiparticle nature has resulted in many interesting optical properties in graphene [3-11]. Graphene absorbs only $2.3 \%$ of the incident light in its pristine form [4]. The optical conductance can, however, be significantly enhanced by cutting graphene into nano-scale ribbon $[5,6]$. The exceptionally strong nonlinear multiple-photon coupling of graphene in the terahertz $(\mathrm{THz})$ frequency regime is another manifestation of the linear energy spectrum [7-9]. Surprisingly, the strong $\mathrm{THz}$ optical nonlinearity is wellpreserved even in its bi-layered form [10] and in the case of a finite bandgap opening [11].

In a graphene superlattice created by applying a KronigPenney potential $[12,13]$, the K-point electrons no longer travels with uniform $v_{\mathrm{F}}$ in all directions. Instead, the group velocity in the direction perpendicular to the periodicity of the Kronig-Penney potential is reduced by a factor of $\lambda$ dependent on the strength and periodicity of the potential. The band structure of the superlattice is no longer circular, but is deformed to an elliptic cone. Such a quasiparticle nature is analogue to a massless Dirac fermion travelling in anisotropic spacetime [12-14]. In topological insulator (TI), the quasiparticle residing in its surface state is also in the massless Dirac form with Fermi velocity approximately half of the graphene [15]. Interestingly, the anisotropic massless Dirac fermion can also be found in the $(2,2,1)$ side-surface state of $\mathrm{Bi}_{2} \mathrm{Se}_{3}$ TI with a rather strong anisotropy of $v_{x}=$ $3.1 \times 10^{5} \mathrm{~m} \mathrm{~s}^{-1}$ and $v_{y}=1.4 \times 10^{5} \mathrm{~m} \mathrm{~s}^{-1}$ [16]. In a Bi square net of $\mathrm{SrMnBi}_{2} \mathrm{TI}$, highly anisotropic Fermi velocity differs by a factor of 8 and was experimentally observed [17].

The effect of anisotropy on the optical response in the terahertz frequency regime is investigated. The optical conductance plays an important role in understanding the electronic transitions and collective excitation of the system [3, $4,18]$. It is directly related to the measurable quantities of absorption coefficient and the transmittance. It is found that 


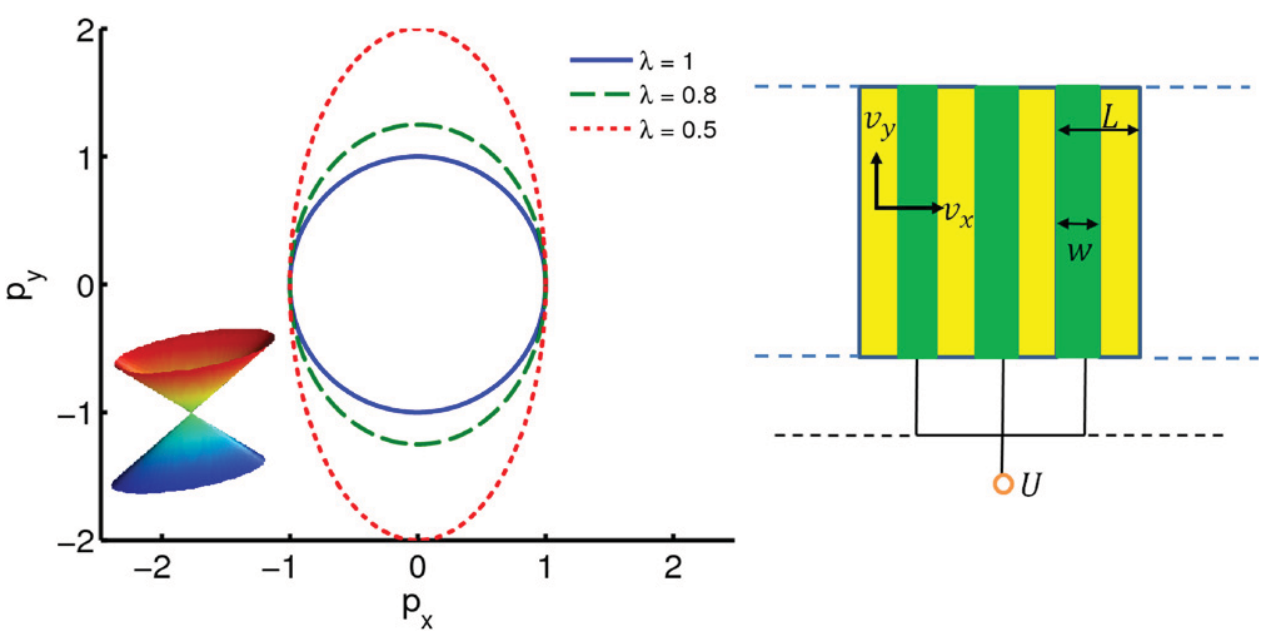

Figure 1. Band structure of graphene superlattice. The elliptic Dirac cone is elongated in the $y$-direction. $L, w$ and $U$ are the superlattice periodicity, potential width and potential height, respectively.

the optical conductance, both linear and nonlinear response, are universally enhanced by the anisotropy when the external field aligns with the superlattice periodicity. Since both linear and nonlinear responses are enhanced by the same amount, the optical nonlinearity (i.e. the relative magnitude between linear and nonlinear responses) is unexpectedly preserved regardless of how strong the band structure anisotropy is. The enhanced optical conductance and the preserved optical nonlinearity reveal that anisotropy has transformed the graphene superlattice into a stronger nonlinear material which produces larger nonlinear optical current than the isotropic case under the same critical electric field strength. Such enhanced optical conductance and well-preserved optical nonlinearity also occurs in gapped graphene in which the quasiparticle is in the form of massive Dirac fermions. The anisotropic massive Dirac fermion is a bizarre quasiparticle not only with nonuniform 'light speed' but also non-uniform mass dependent on the propagation direction. The results suggest that the enhanced electron-single-photon and electron-multiplephoton couplings are a universal feature of relativistic Dirac fermions of both massless or massive types, and the band structure isotropy is not a pre-requisite for the strong optical nonlinearity in graphene.

\section{Theory}

The anisotropy can be modelled by defining an anisotropy parameter, $\lambda$, which modifies the $y$-direction group velocity by $v_{y}=\lambda v_{\mathrm{F}}$ where $v_{\mathrm{F}}=10^{6} \mathrm{~m} \mathrm{~s}^{-1}$ is the Fermi velocity, and the anisotropy parameter is continuously tunable, $0 \leqslant \lambda \leqslant 1$, by varying the superlattice periodicity $L$, potential width $w$ and potential height $U$ (figure 1 ) $[12,13]$. The graphene superlattice Hamiltonian is written as $\hat{\mathcal{H}}=\sigma_{x} p_{x}+\lambda \sigma_{y} p_{y}$ where $\sigma$ 's are the Pauli spin matrices and the $\lambda$ term has created the desired anisotropy in the $y$-direction. The energy dispersion is given as $\varepsilon_{s}(p, \theta)=s v_{\mathrm{F}} p \sqrt{\cos ^{2} \theta+\lambda^{2} \sin ^{2} \theta}$ where $s= \pm 1$ denotes electron and hole state. The group velocity in the $\theta$ direction is given as $v(\theta)=v_{\mathrm{F}} \sqrt{\cos ^{2} \theta+\lambda^{2} \sin ^{2} \theta}$ which gives the expected $x$ - and $y$-components of $v(\theta=0)=v_{\mathrm{F}}=v_{x}$ and $v(\theta=\pi / 2)=\lambda v_{\mathrm{F}}=v_{y}$. The eigenfunction is given as $\psi_{0}(s, p)=\frac{1}{\sqrt{2}}\left(1, v_{\mathrm{F}}\left(p_{x}+\mathrm{i} \lambda p_{y}\right) / \varepsilon_{s}\right)^{\mathrm{T}}$ where $\mathrm{T}$ denotes transpose. The band structure is plotted in figure 1 . It can be seen that due to the reduced group velocity in the $y$-direction, the conic Dirac cone is elongated in the $y$-direction, forming an anisotropic elliptic Dirac cone. When an external field $\boldsymbol{E}=\boldsymbol{E}_{0} \mathrm{e}^{\mathrm{i} \omega t}$ is applied along the $x$-direction, the quasiparticle is minimally coupled to the photon according to $p_{x} \rightarrow p_{x}-e A$ where $\boldsymbol{E}=-\partial \boldsymbol{A} / \partial t$. The Hamiltonian is then given by

$$
\hat{\mathcal{H}}=v_{\mathrm{F}}\left[\begin{array}{cc}
0 & \tilde{p}_{-}-e A \\
\tilde{p}_{+}-e A & 0
\end{array}\right],
$$

where $\tilde{p}_{ \pm}=p_{x} \pm \lambda \mathrm{i} p_{y}$. The perturbed two-spinor wavefunction (consider only the $s=1$ branch) can be expressed in terms of an infinite sum

$$
\psi(p)=\sum_{n}\left[\begin{array}{l}
a_{n} \\
b_{n}
\end{array}\right] \mathrm{e}^{\mathrm{i}\left(\frac{\varepsilon}{\hbar}-n \omega\right) t},
$$

where $\varepsilon=\varepsilon_{s=1}$. Each $n$ th-order spinors $a_{n}$ and $b_{n}$ represents a particular $n$-photon process. Equations (1) and (2) can be readily solved via Schrodinger's equation i $\hbar \partial \psi(p) / \partial t=$ $\hat{\mathcal{H}} \psi(p)$. This yields the following recursion equations linking $n$ th-order spinors with $(n-1)$ th spinor components:

$$
\begin{aligned}
& (\varepsilon-n \hbar \omega) a_{n}=v_{\mathrm{F}} \tilde{p}_{-} b_{n}+\frac{e E_{0} v_{\mathrm{F}}}{\mathrm{i} \omega} b_{n-1}, \\
& (\varepsilon-n \hbar \omega) b_{n}=v_{\mathrm{F}} \tilde{p}_{+} a_{n}+\frac{e E_{0} v_{\mathrm{F}}}{\mathrm{i} \omega} a_{n-1} .
\end{aligned}
$$

By recursively applying equation (3), any arbitrary $n$ th-order spinors can be constructed. When $n=0$, the zeroth-order spinor $a_{0}$ and $b_{0}$ agrees with the zero-photon unperturbed wavefunction. The $n$ th-order current is proportional to the $E_{0}^{n}$ and the convergence of equation (3) is determined by intensity and frequency of the applied field. The total current density in the $x$-direction can then be obtained from $J_{x}^{(n)}=$ $1 /(2 \pi \hbar)^{2} \int \psi_{n}^{\dagger} \hat{v}_{x} \psi_{n} \mathrm{~d} \boldsymbol{p}^{2} \mathcal{N}(\varepsilon)$ where the velocity operator is given as $\hat{v}_{x}=\partial \hat{\mathcal{H}} / \partial p_{x}$ and $\mathcal{N}(\varepsilon)=f(-\varepsilon)-f(\varepsilon)=$ $\tanh (\beta \varepsilon / 2)$ is a temperature factor describing the availability 


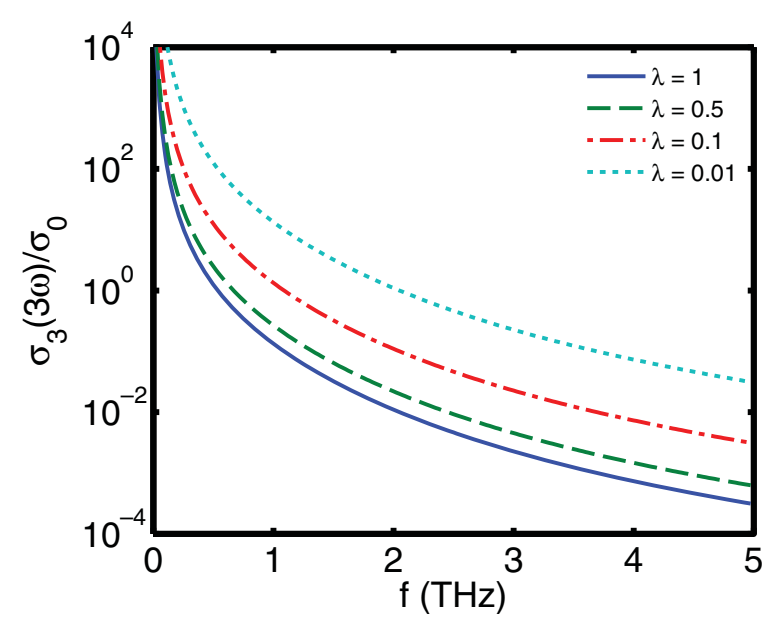

Figure 2. The frequency dependence of $\sigma_{3}(3 \omega)$ at $E_{0}=1000 \mathrm{~V} \mathrm{~cm}^{-1}$ and $300 \mathrm{~K}$.

of the $s=-1$ carrier (hence the $+f(-\varepsilon)$ term) and the occupancy of the $s=+1$ carrier (hence the $-f(\varepsilon)$ term) at finite temperature. $f(\epsilon)=\left[\exp \left(\epsilon / k_{\mathrm{B}} T\right)+1\right]^{-1}$ is the Fermi-Dirac distribution function for an intrinsic graphene and $\beta=\left(k_{\mathrm{B}} T\right)^{-1}$ is the inverse temperature. By selecting the terms containing $n$th order in the external field $\boldsymbol{E}$, the linear $(n=1)$ and third-order nonlinear $(n=3)$ optical responses are derived. The second-order $(n=2)$ response is zero due to time-reversal symmetry. The linear optical conductivity of the graphene superlattice is given as

$$
\sigma_{1}(\omega)=\frac{1}{\lambda} \frac{e^{2}}{4 \hbar} \mathcal{N}\left(\frac{\hbar \omega}{2}\right)
$$

At $T=0 \mathrm{~K}$, the linear response is reduced to the well known result $\sigma_{1}(\omega)=\sigma_{0}$ in the isotropic case $\lambda=1$. The third-order nonlinear optical conductivities are given as

$\sigma_{3}(\omega)=\frac{2 \mathcal{E}^{2}}{\lambda} \mathcal{N}\left(\frac{\hbar \omega}{2}\right)$,

$\sigma_{3}(3 \omega)=\frac{\mathcal{E}^{2}}{\lambda}\left[\frac{13}{48} \mathcal{N}\left(\frac{\hbar \omega}{2}\right)-\frac{2}{3} \mathcal{N}(\hbar \omega)+\frac{15}{16} \mathcal{N}\left(\frac{3 \hbar \omega}{2}\right)\right]$,

where $\mathcal{E} \equiv\left(\sigma_{0}^{1 / 2} E_{0} v_{\mathrm{F}} e\right) / \hbar \omega^{2}$. The third-order nonlinear response is made up of two components: the single-frequency (SF) term $\sigma_{3}(\omega)$ corresponding to the simultaneous absorption of two photons and the immediate emission of one photon; and the triple-frequency (TF) term $\sigma_{3}(3 \omega)$ corresponding to the simultaneous absorption of three photons. In figure $2, \sigma_{3}(3 \omega)$ at $T=300 \mathrm{~K}$ and $E_{0}=1000 \mathrm{~V} \mathrm{~cm}^{-1}$ are plotted for different $\lambda$. The practically useful frequency-tripling effect represented by $\sigma_{3}(3 \omega)$ is up-shifted in the graphene superlattice due to the Dirac fermions anisotropy. Interestingly, $\sigma_{1}(\omega), \sigma_{3}(\omega)$ and $\sigma_{3}(3 \omega)$ are all universally enhanced by a factor of $1 / \lambda$. For $\lambda=0.1$, which can be achieved by applying a spatial period of $L \approx 20 \mathrm{~nm}$, potential width of $w=10 \mathrm{~nm}$ and potential height of $U=0.3 \mathrm{eV}$, the total optical conductance is enhanced by 10 times. In the extremely anisotropic case of $\lambda=0.01$, which can be achieved by $L \approx 25 \mathrm{~nm}, w=10 \mathrm{~nm}$ and $U=0.3 \mathrm{eV}$ [12], 100 times amplification is achieved. The
$1 / \lambda$ enhanced optical conductance is quite a surprising result. Intuitively, one might expect a reduced optical response in the anisotropic case since the $y$-component of the group velocity $v_{y}=\lambda v_{\mathrm{F}}$ is reduced by a factor of $\lambda$ and the resulting 'slower' charge carrier should degrade the optical current. This is, however, not the complete picture since $\boldsymbol{E}$ is directed along the $x$-direction and the $x$-directional optical response is only minimally affected by the reduced $y$-directional group velocity $v_{y}=\lambda v_{\mathrm{F}}$. On the other hand, when $\lambda<1$ the $p_{y}$ components in a equi-energy slice actually becomes larger in comparison with the isotropic Fermi velocity case because of the smaller slope (or equivalently the reduced $v_{y}$ ) in the $y$-direction (see figure 1). The overall larger momentum of the charge carrier across an equi-energy surface is the underlying reason for the anisotropy-induced enhancement of the optical conductance in the Kronig-Penney type graphene superlattice. As the anisotropy increases, i.e. $\lambda \rightarrow 0$, the band structure becomes more $y$-directionally elongated across an equi-energy surface and this results in the $1 / \lambda$ dependence.

The optical nonlinearity of a material is defined as the relative magnitude between the linear and nonlinear optical responses. A particularly useful quantity commonly used is the critical field strength $E_{\mathrm{c}}$ which is defined as the electric field strength required to create nonlinear conductivities which are as strong as the linear one, i.e. when $\sigma_{3}=\sigma_{1}$. A small $E_{\mathrm{c}}$ directly indicates strong optical nonlinearity. It is interesting to see that in the graphene superlattice the $E_{\mathrm{c}}$ remains the same regardless of the level of anisotropy since both linear and nonlinear responses are enhanced by the same factor of $1 / \lambda$. Therefore, just like normal graphene, the graphene superlattice is also an exceptionally strong nonlinear material with $E_{\mathrm{c}} \approx$ $10^{3} \mathrm{~V} \mathrm{~cm}^{-1}$ for up to room temperature at $f=1 \mathrm{THz}$. It can be concluded that the strong optical nonlinearity observed in normal graphene and graphene superlattice is a general feature of the relativistic behaviour of the quasiparticle. The band structure isotropy is not necessarily required to achieve the strong optical nonlinearity. As long as the quasiparticle energy dispersion maintains its linear form, the strong optical nonlinearity is always guaranteed and is well protected from any band structure anisotropy. The total integrated optical spectra is given as $\Sigma(\lambda)=\int \sigma(\omega, \lambda) \mathrm{d} \omega$ and it can be immediately seen that the total nonlinear conductance is increased by a factor $1 / \lambda$ for all of the $\mathrm{THz}$ frequency regime, as shown in figure 3 . Although the graphene superlattice is equally advantageous as normal graphene in terms of the smallness of $E_{\mathrm{c}}$, the $1 / \lambda$ increased total conductance indicates that the nonlinear optical current output of the graphene superlattice is still larger than that of the normal graphene at a given electric field strength. This suggests the improved $\mathrm{THz}$ photon detection and $\mathrm{THz}$ frequency up-conversion in the graphene superlattice which are potentially useful in the development of graphene-based $\mathrm{THz}$ optical device.

When $\boldsymbol{E}$ is placed along the $y$-direction, $j_{y}$ becomes finite while $j_{x}$ becomes strictly zero since the quasiparticles do not gain any excess momentum in the $x$-direction. In this case, the perturbed Hamiltonian is given as $\hat{\mathcal{H}}=\sigma_{x} p_{x}+\lambda \sigma_{y}\left(p_{y}-e A\right)$ and the velocity operator becomes $\hat{v}_{y}=v_{\mathrm{F}} \lambda \sigma_{y}$. It can be shown that the $y$-directional conductivities are of the form

$$
\sigma_{n}^{(y)}(\mathrm{j} \omega)=\lambda^{2} \sigma_{n}(\mathrm{j} \omega),
$$




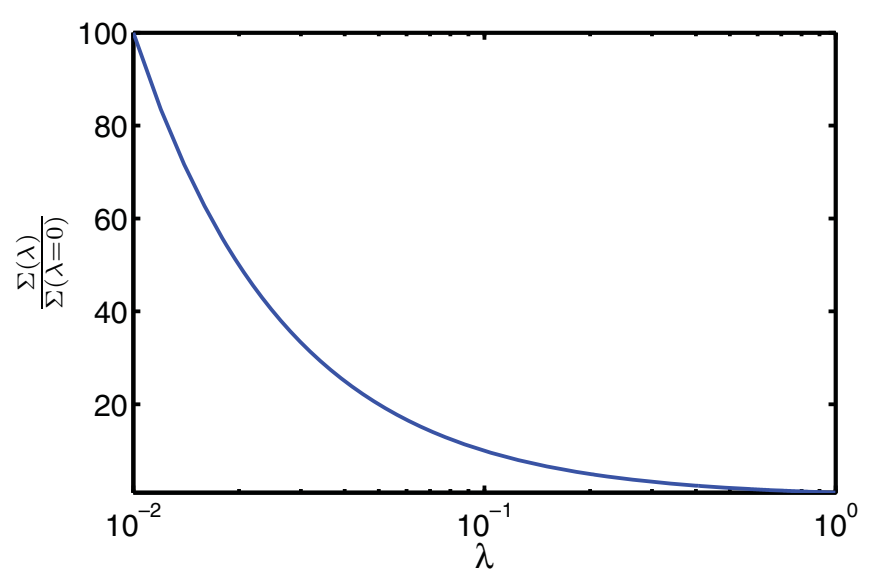

Figure 3. The $\lambda$-dependence of the integrated anisotropic conductivity (normalized by integrated isotropic conductivity).

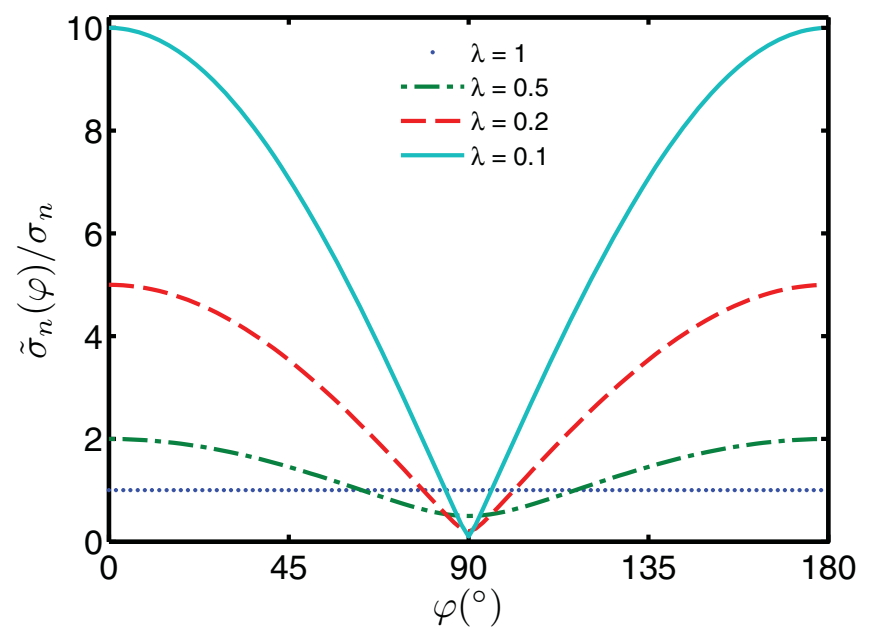

Figure 4. $\varphi$-dependence of the total conductivity. Different behaviour occurs at the vicinity of $\varphi=\pi / 2$ when $\propto \lambda$ behaviour dominates over the $1 / \lambda$ behaviour.

where $n=1,3$ and $j=1,3$. The $\lambda^{2}$ factor originates from $\hat{v}_{y}$ and the $y$-momentum coupling $\lambda\left(p_{y}-e A\right)$. Unlike the $x$-direction case, $\boldsymbol{E}$ along the $y$-direction has resulted in a anisotropy dependence of $\sigma^{(y)}(\omega) \propto \lambda$. The optical conductance is hence reduced by a factor of $\lambda$. This is an obvious consequence since the reduced $y$-directional group velocity $v_{y}$ has a more profound effect on the $y$-directional optical response when $\boldsymbol{E}$ is aligned in the $y$-direction. In a more general case when $\boldsymbol{E}$ is in any angle $\varphi$ as measured from the $x$-axis, the combine effect of the $x$-directional $\sigma$ and $\sigma^{(y)}$ can be investigated by defining the total conductivity, measured along the $\varphi$-direction, as $\tilde{\sigma}(\varphi)^{2}=\sigma^{2}+\left(\sigma^{(y)}\right)^{2}$ :

$$
\tilde{\sigma}_{n}(\varphi, \mathrm{j} \omega)=\left(\frac{\cos ^{2}(\varphi)}{\lambda^{2}}+\lambda^{2} \sin ^{2}(\varphi)\right)^{1 / 2} \sigma_{n}(\mathrm{j} \omega) .
$$

The angular dependence of $\tilde{\sigma}_{n}(\varphi, \mathrm{j} \omega)$ is plotted in figure 4. For notational simplicity, $(\mathrm{j} \omega)$ in the argument is suppressed. The $\tilde{\sigma}_{n}$ has a very strong $\varphi$-dependence especially in the highly anisotropic case. For $\lambda=0.1, \tilde{\sigma}_{n} / \sigma_{n}$ varies between 10 and 0.1 with $\varphi$. Maximum conductance occurs at $\varphi=0, \pi$ when $\boldsymbol{E}$ is along the $x$-axis causing the $1 / \lambda$ enhancement to be dominant.
For most of the $\varphi, \sigma_{n}(\varphi)$ is larger for stronger anisotropy, except a contrasting behaviour of the reduced conductance at higher anisotropy occurring in the vicinity of $\varphi=\pi / 2$ when $\sigma_{n}^{(y)}$ becomes a dominant contributor and the $\propto \lambda$ dependence dominates over the $1 / \lambda$ dependence.

Since we only include the third order term in our result, we comment briefly on the relative importance of the higher order nonlinear terms. The $n$ th-order conductance is proportional to a dimensionless parameter $Z=\left(e E_{0} v_{\mathrm{F}} / \hbar \omega^{2}\right)^{n-1}$ and an overlap integral of eigenstates of different orders $\left\langle\phi_{n-m} \mid \phi_{m}\right\rangle$. Because the overlap integral decreases very rapidly with $n$, the third-order nonlinear effect persists for $Z>1$ while the fifth-order term is negligible. At frequency around $1 \mathrm{THz}$, the critical field (the field at which the third-order current equals the linear current) is around $2000 \mathrm{~V} \mathrm{~cm}^{-1}$ [7]. For $V_{\mathrm{F}}=10^{6} \mathrm{~m} \mathrm{~s}^{-1}, \omega=1 \mathrm{THz}$, and $E_{0}=2000 \mathrm{~V} \mathrm{~cm}^{-1}$, the resulting $Z=50$. At this value of $Z$, the third-order current equals approximately the linear current [7], but the fifth-order current is about $10^{-5}$ of the linear current, totally negligible [21].

\section{Anisotropic massive Dirac fermions}

The effect of Dirac fermion anisotropy on the optical response is further generalized to the case of massive Dirac fermions which can be found in a gapped graphene. An asymmetry between the two equivalent sublattices in the unit cell of graphene transforms the low-energy quasiparticles into massive Dirac fermions which are described by the finite-mass Dirac equation $\hat{\mathcal{H}}_{\Delta}=\sigma_{x} p_{x}+\lambda \sigma_{y} p_{y}+(\Delta / 2) \sigma_{z}$ where $\Delta / 2$ is the on-site energy difference between the two sublattices. The sublattice asymmetry can be achieved by selectively 'doping' an hydrogen atom on one of the sublattices or by placing graphene on top of a suitable substrate such as $\mathrm{SiC}[19,20]$. We choose a typical bandgap value of $\Delta=0.03 \mathrm{eV}$ [19]. The energy dispersion is given as $\varepsilon_{s}(p, \theta)=s \sqrt{v_{\Delta}^{2} p^{2}+(\Delta / 2)^{2}}$, which indicates a bandgap opening of $\Delta$ or a 'mass generation' of $m=\Delta /\left(2 v_{\Delta}\right)^{2}$ of the quasiparticle, where $v_{\Delta}=v_{\mathrm{F}}\left(\cos ^{2} \theta+\right.$ $\left.\lambda^{2} \sin ^{2} \theta\right)$. The anisotropic massive Dirac fermions are a rather exotic class of quasiparticles which not only have a 'light speed' anisotropically dependent on $\theta$ but also have a non-uniform 'mass' that varies with $\theta$. Following similar procedures, the linear and nonlinear optical conductivities of gapped graphene with band structure anisotropy are given as

$$
\begin{gathered}
\sigma_{1}^{\Delta}(\omega)=\frac{\sigma_{0}}{\lambda}\left[1+\tilde{\Delta}^{2}\right] \mathcal{N}\left(\frac{\hbar \omega}{2}\right) \Theta(\hbar \omega-\Delta), \\
\sigma_{3}^{\Delta}(3 \omega)=\frac{\mathcal{E}^{2}}{\lambda} \frac{2}{2+\tilde{\Delta}} X_{0} \mathcal{N}(\hbar \omega) \Theta(\hbar \omega-\Delta), \\
\sigma_{3}^{\Delta}(3 \omega)=\frac{\mathcal{E}^{2}}{\lambda} Y \Theta(3 \hbar \omega-\Delta),
\end{gathered}
$$

where $X_{0}=2+\tilde{\Delta}+\tilde{\Delta}^{2}+\tilde{\Delta}^{3} / 2-3 \tilde{\Delta}^{4} / 8-3 \tilde{\Delta}^{5} / 16, Y=$ $X_{1} \mathcal{N}(\hbar \omega / 2)+X_{2} \mathcal{N}(\hbar \omega)+X_{3} \mathcal{N}(3 \hbar \omega / 4), X_{1}=(1 / 48)[13+$ $\left.2 \tilde{\Delta}^{2}+\tilde{\Delta}^{4}\right], X_{2}=-(1 / 3)\left[2-\tilde{\Delta}^{2}+\tilde{\Delta}^{4} / 8\right], X_{3}=(1 / 48)[45-$ $\left.14 \tilde{\Delta}^{2}+\tilde{\Delta}^{4}\right]$ and $\tilde{\Delta}=\Delta / \hbar \omega$.

Interestingly, optical response in gapped graphene is similarly tuned-up by a factor of $1 / \lambda$. This indicates that 


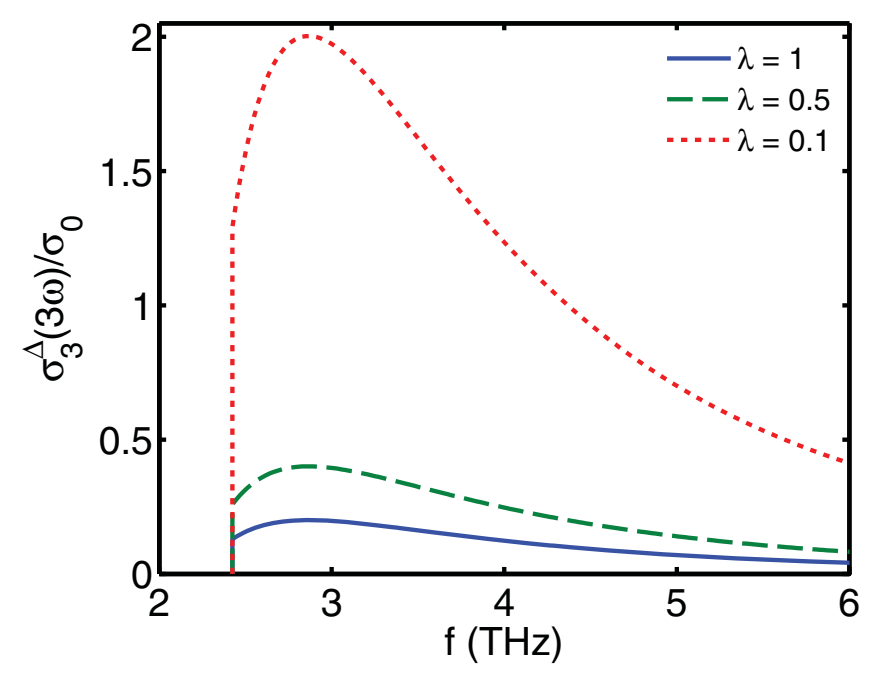

Figure 5. Anisotropic gapped graphene frequency-tripling conductivity at $300 \mathrm{~K}$ and $E_{0}=3400 \mathrm{~V} \mathrm{~cm}^{-1}$ and $\Delta=0.03 \mathrm{eV}$.

the anisotropy-enhanced optical conductance observed in the graphene superlattice is not a phenomena unique only to the massless Dirac fermions, but is in fact a universal feature for both massless and massive relativistic Dirac fermions. Similarly, the $E_{\mathrm{c}}$ remains the same regardless of the magnitude of $\lambda$. The well-preserved optical nonlinearity in anisotropic massless Dirac fermions is therefore also general to anisotropic massive Dirac fermions. The linear conductivity has an onset vertical conductance edge of $2 \sigma_{0}$ at $\hbar \omega=\Delta$. In the anisotropy case of $\lambda=0.1$, this vertical conductance edge is further enhanced. At the sub-gap frequency regime of $\Delta / 3<\hbar \omega<\Delta$, the three-photon process $\sigma_{3}^{\Delta}(3 \omega)$ is the only permissible response due to the virtual of energy conservation. In figure $5, \sigma_{3}^{\Delta}(3 \omega)$ is plotted at $300 \mathrm{~K}, E_{\mathrm{c}}=3400 \mathrm{~V} \mathrm{~cm}^{-1}$ and $\Delta=0.03 \mathrm{eV}$ in the sub-gap frequency regime. In this regime, the $1 / \lambda$-amplification of $\sigma_{3}^{\Delta}(3 \omega)$ is the sole effect and the linear conductance and SF $\sigma_{3}(\omega)$ transitions are strictly forbidden. This suggests that anisotropy-enhanced 'clean' frequency upconversion, i.e. frequency-tripling current is the only output, can be achieved in gapped graphene with the anisotropic band structure.

\section{Conclusion}

In conclusion, the anisotropic Dirac fermion in the graphene superlattice tunes up the total optical conductance while maintaining the same critical electric field. Such anisotropyinduced optical conductance enhancement also occurs in anisotropic gapped graphene. This suggests that the enhanced electron-photon couplings in the presence of anisotropy is a general feature of the relativistic nature of the Dirac fermions in both massless and massive form. Optical nonlinearity is perfectly protected from band structure anisotropy. As a weak sinusoidal term can be added to a graphene via holographic illumination [22] or by patterning the substrate, possible experimental verification of our results could be performed with direct measurement of the optical conductance of such a system.

\section{References}

[1] Novoselov K A, Geim A K, Morozov S V, Jiang D, Zhang Y, Dubonos S V, Grigorieva I V and Firsov A A 2004 Science 306 666-9

[2] Geim A K and Novoselov K S 2007 Nature Mater. 6 183-91

[3] Zhang C, Chen L and Ma Z 2008 Phys. Rev. B 77 241402(R)

[4] Nair R R, Blake P, Grigorenko A N, Novoselov K S, Booth T J, Stauber T, Peres N M R and Geim A K Science 3201308

[5] Liu J, Wright A R, Zhang C and Ma Z 2008 Appl. Phys. Lett. 93041106

[6] Wright A R, Cao J C and Zhang C 2009 Phys. Rev. Lett. 103207401

[7] Wright A R, Xu X G, Cao J C and Zhang C 2009 Appl. Phys. Lett. 95072101

[8] Mikhailov S A 2007 Europhys. Lett. 7927002

[9] Shareef S, Ang Y S and Zhang C 2012 J. Opt. Soc. Am. B 29 274-9

[10] Ang Y S, Sultan S and Zhang C 2010 Appl. Phys. Lett. 97243110

[11] Ang Y S and Zhang C 2011 Appl. Phys. Lett. 98042107

[12] Park C H, Yang L, Son Y-W, Cohen M L and Louie S G 2008 Nature Phys. 4 213-17

[13] Park C H, Yang L, Son Y-W, Cohen M L and Louie S G 2008 Phys. Rev. Lett. 101126804

[14] Edwards W F 1963 Am. J. Phys. 31482

[15] Hasan M Z and Kane C L 2010 Rev. Mod. Phys. 82 3045-67

[16] Moon C Y, Han J, Lee H and Choi H J 2011 Phys. Rev. B 84195425

[17] Park J et al 2011 Phys. Rev. Lett. 107126402

[18] Zhang C 2002 Phys. Rev. B 66081105 (R)

[19] Chen L, Ma Z and Zhang C 2010 Appl. Phys. Lett. 96023107

[20] Zhou S Y, Gweon G H, Fedorov A V, First P N, de Heer W A, Lee D H, Guinea F, Neto A H C and Lanzara A 2007 Nature Mater. 6 770-5

[21] Xu X G and Cao J C 2010 Mod. Phys. Lett. B 242243

[22] Weiss D, Zhang C, Gerhardts R R, Klitzing K v and Weimann G 1989 Phys. Rev. B 3913020 